\title{
Identification of molecular signatures involved in radiation-induced lung fibrosis
}

\author{
Hee Jin ${ }^{1} \cdot$ Ga-Young Kang ${ }^{1} \cdot$ Seulgi Jeon ${ }^{1} \cdot J_{\text {Jin-Mo Kim }}{ }^{2} \cdot$ You Na Park ${ }^{1} \cdot$ Jaeho Cho $^{2} \cdot$ Yun-Sil Lee $^{1}$
}

Received: 30 May 2018 / Revised: 30 October 2018 / Accepted: 31 October 2018 /Published online: 7 November 2018

(C) The Author(s) 2018

\begin{abstract}
In radiotherapy, radiation (IR)-induced lung fibrosis has severe and dose-limiting side effects. To elucidate the molecular effects of IR fibrosis, we examined the fibrosis process in irradiated mouse lung tissues. High focal IR (90 Gy) was exposed to a 3-mm volume of the left lung in C57BL6 mice. In the diffused irradiation, $20 \mathrm{~Gy}$ dose delivered with a 7-mm collimator almost covered the entire left lung. Histological examination for lung tissues of both irradiated and neighboring regions was done for 4 weeks after irradiation. Long-term effects ( 12 months) of 20Gy IR were compared on a diffuse region of the left lung and non-irradiated right lung. Fibrosis was initiated as early as 2 weeks after IR in the irradiated lung region and neighboring region. Upregulation of gtsel in both 90Gy-irradiated and neighboring regions was observed. Upregulation of $f g l 1$ in both 20Gy diffused irradiated and non-irradiated lungs was identified. When gtsel or $f l g 1$ was knock-downed, TGF $\beta$ or IR-induced epithelial-mesenchymal transition was inhibited, accompanied with the inhibition of cellular migration, suggesting fibrosis responsible genes. Immunofluorescence analysis using mouse fibrotic lung tissues suggested that fibrotic regions showed increased expressions of Gtsel and Fgll, indicating novel molecular signatures of gtseland fgll for IR-induced lung fibrosis. Even though their molecular mechanisms and IR doses or irradiated volumes for lung fibrosis may be different, these genes may be novel targets for understanding IR-induced lung fibrosis and in treatment strategies.
\end{abstract}

\section{Key messages}

- Upregulation of gtsel by $90 \mathrm{~Gy}$ focal irradiation and upregulation of $f g l l$ by $20 \mathrm{~Gy}$ diffused irradiation are identified in mouse lung fibrosis model.

- Gtse1 and Fgl1 are involved in radiation or TGF $\beta$-induced epithelial-mesenchymal transition.

- Radiation-induced fibrotic regions of mouse lungs showed increased expressions of Gtse1 and Fgl1.

- Gtse1 and Fgl1 are suggested to be novel targets for radiation-induced lung fibrosis.

Keywords Irradiation $\cdot$ Lung fibrosis $\cdot$ Epithelial-mesenchymal transition $\cdot g t s e l \cdot f g l l$

Hee Jin and Ga-Young Kang contributed equally to this work.

Electronic supplementary material The online version of this article (https://doi.org/10.1007/s00109-018-1715-9) contains supplementary material, which is available to authorized users.

Yun-Sil Lee

yslee0425@ewha.ac.kr

1 Graduate School of Pharmaceutical Sciences, Ewha Womans University, Seoul 120-750, South Korea

2 Department of Radiation Oncology, Yonsei University Health System, Seoul 120-749, South Korea

\section{Introduction}

Radiation (IR)-induced pulmonary fibrosis is a frequently occurred complication from radiotherapy threatening the health and life of patients $[1,2]$. The time of onset and severity of lung fibrosis after radiotherapy depend on many factors, including the volume of irradiated parenchyma, dose of absorbed IR, and number of fractions [3-5]. Despite clinical evidence of lung injury due to IR, the molecular mechanisms underlying the effect of IR, especially those focusing on lung fibrosis, have not been clearly identified.

The goal of high-dose per fraction irradiation (HDFR), such as stereotactic body radiotherapy (SBRT), is to 
administer a markedly higher dose to the treatment target volume without damaging the surrounding normal tissue. The targeting accuracy of IR to the tumor by SBRT produces reduced damage to surrounding normal tissue. It is feasible to use HDFR without inducing significant acute and late IR-induced toxicity with SBRT. However, there are still concerns of late toxicity following high-dose treatment. Because large doses of IR are delivered to the target volume, adjacent normal tissue damage may not compromise the benefits of HDFR for better control because underlying radiobiological mechanisms of damage by large dose per fractions remain the same.

We have previously developed a mouse model simulating clinical SBRT and have used the model to validate the induction of lung fibrosis by high-dose IR [6]. We also attempted to understand the biological changes occurring in the process of lung tissue damage including cell death after SBRT. In addition, we identified responsible genes and proteins for IR-induced lung damage [7]. In this study, to identify molecular signatures for IR-induced lung fibrosis, we further examined the fibrosis process at 4 weeks in non-irradiated neighboring regions as well as irradiated region from mouse lung tissues after HDFR (90 Gy). The regimen was similar to that used for human therapy reflecting the understanding of the clinically related HDFR-mediated normal cell damage like fibrosis. Long-term effects (12 months) on diffuse region of the left lung and non-irradiated right lung after 20Gy IR which dose ranges (15$20 \mathrm{~Gy}$ ) are frequently used as IR-induced lung fibrosis model [8-11] were also compared.

\section{Materials and methods}

\section{Antibody and reagents}

Protein levels were detected using commercial antibodies to the following: GTSE1 (Thermo Scientific, Waltham, MA, USA or Sigma-Aldrich, St. Louis, MO, USA), FGL1 (Thermo Scientific or Sigma-Aldrich); $\alpha$ SMA (Sigma-Aldrich); Twist (Abcam, Cambridge, UK), Matrix metallopeptidase 12 (MMP12; Abcam), Gapdh (Abcam); Fibronectin (BD Biosciences, Santa Clara, CA, USA); and Twist (Santa Cruz Biotechnology, Santa Cruz, CA, USA), $\beta$-Actin (Santa Cruz). Predesigned small interfering (si) RNAs for human gtsel and fgll and a negative control siRNA were purchased from Bioneer (Daejeon, Republic of Korea). Transforming growth factor-beta 1 (TGF- $\beta 1$ ) was purchased from BD Biosciences.

\section{Cell culture and transfection}

A549 human lung adenocarcinoma cells and L132 human pulmonary epithelial cells were supplied from American Type Culture Collection (ATCC, Manassas, VA, USA). All cells were cultured in RPMI medium supplemented with $10 \%$ fetal bovine serum and $1 \%$ penicillinstreptomycin at $37{ }^{\circ} \mathrm{C}$ in humidified $5 \% \mathrm{CO}_{2}$ incubator. Transient transfection of all cell types used Lipofectamine 2000 (Invitrogen, Carlsbad, CA, USA) as instructed by the manufacturer.

\section{Irradiation}

Five adult male C57BL/6 mice (Central Lab Animal Inc., Seoul, Korea) were housed per each cage (10week-old). Total 60 male mice were randomly divided into four groups. Two groups were for 90Gy experiment (control and 90Gy focal irradiated groups) and two groups were for 20Gy experiment (control and 20Gy irradiated groups). As the clinical SBRT condition, we selected a 3-mm collimator to administer a 90Gy dose to the central area of the left lung. For low-dose diffused irradiation conditions, we delivered a 20Gy dose with a 7-mm collimator, which almost covered the entire left lung. In the mice that underwent 90Gy irradiation, focal irradiated and neighboring tissues were separately isolated (Supplementary Fig. S1b). In the mice that underwent 20Gy irradiation, the whole left lungs were used for irradiated tissues and non-irradiated right lungs were also used. Control lungs were isolated from the age-matched control mice. Radiation was administered with an X-RAD 320 (Precision, North Branford, CT) equipped with a collimator system that consisted of 5-cm-thick copper to focus the radiation beams. X-ray beam dose rate was $19.7 \mathrm{cGy} / \mathrm{s}$. Detailed methods are described previously [12]. In the case of cells, they were exposed to $\gamma$-rays using a ${ }^{137} \mathrm{Cs} \gamma$-ray source (Atomic Energy of Canada) with a dose rate of $3.81 \mathrm{~Gy} / \mathrm{min}$.

\section{Microarray experiment, RNA isolation, and quantitative RT-PCR (qPCR)}

Total RNA from the mouse lung tissues was prepared using the Easy-SpinTM total RNA extraction kit according to the manufacturer's instructions (iNtRON Biotechnology, Seoul, Republic of Korea). RNAs from 3 mice at each time point were pooled to exclude experimental bias. Detailed methods are described in the Supplementary information. For quantitative RT-PCR, total RNA was isolated from lung tissues of experimental mice or cells at each time points post radiation exposure using TRIzol ${ }^{\circledR}$ reagent (Qiazen, 
Valencia, CA, USA). RNA purity and concentration were measured with a Nanodrop. RNA was reverse transcribed using a ReverTra Ace® qPCR RT Kit (TOYOBO, Kita-ku, Osaka, Japan) following the manufacturer's protocol and PCR was performed to assess expression of the candidate genes using primers designed for mouse mRNA sequences. Also, the expression of mRNAs was assessed by real-time PCR using SYBR Green PCR Master Mix kit (Invitrogen, Carlsbad, CA, USA) with an ABI 7300 real-time PCR thermal cycler (Applied Biosystems, Foster City, CA, USA). The $2^{-\Delta \Delta C t}$ method could be used to analyze the relative changes in gene expression from real-time quantitative PCR experiments. The total reaction volume is $20 \mu \mathrm{L}$. Reaction conditions started with enzyme activation at $95{ }^{\circ} \mathrm{C}$ for $10 \mathrm{~min}$, followed by 40 cycle of $95{ }^{\circ} \mathrm{C}$ for $15 \mathrm{~s}, 58^{\circ} \mathrm{C}$ for $30 \mathrm{~s}$, and $72^{\circ} \mathrm{C}$ for $45 \mathrm{~s}$. Primer sequences for qRT-PCR are listed in Supplementary Table S1.

\section{Immunoblotting}

For immunoblotting, cells were lysed with immune precipitation buffer. Protein concentration was determined by the Bradford method (Bio-Rad, Hercules, CA, USA). The samples were boiled for $5 \mathrm{~min}$ and an equal amount of protein was analyzed by SDS-PAGE (6-15\%) using standard conditions. HRP activity was measured using enhanced chemiluminescence (EzWestLumi, Taito-ku, Tokyo, Japan). Protein band intensity was visualized on ChemiDoc (Bio-Rad) and quantified using Image J software 1.45 (National Institutes of Health, Bethesda, MD, USA).

\section{Histological and immunohistochemical analysis}

Lung tissues were formalin-fixed, paraffin-embedded, and sections were prepared for standard hematoxylin/ eosin (H\&E) staining, Masson trichrome (SigmaAldrich) collagen staining, and picro-sirius red (American MasterTech Scientific, Lodi, CA, USA) staining [13].

\section{Wound healing assay}

For monolayer wound-healing assays, cells were plated in a 60-mm dish and at $100 \%$ confluence, two parallel wounds of $1 \mathrm{~mm}$ were made using a SPLScar (SPL, Gyeonggi-do, Korea). Wound size after 0 , and $48 \mathrm{~h}$ was measured using a light microscope (Cal Zeiss, Oberkochen, Germany) in three independent experiments.

\section{Immunofluorescence}

Cells were fixed with $4 \%$ paraformaldehyde followed by permeabilization with $0.1 \%$ sodium citrate plus $0.1 \%$
Triton X-100. For dual immunofluorescence staining, cells were incubated with Fibronectin $(1: 200)$ or Twist $(1: 200)$ and GTSE1 (1:100) or FGL1 (1:100) antibodies for $1 \mathrm{~h}$ at room temperature. The cells were incubated with Alexa 568-labeled anti-rabbit (1:1000) and Alexa 488-labeled anti-mouse (1:1000) secondary antibodies. For lung tissue immunofluorescence assays, deparaffinized slides were boiled in $0.1 \mathrm{M}$ citrate buffer ( $\mathrm{pH}$ 6.0) for $20 \mathrm{~min}$ and slides were co-immunostained with $\alpha$-SMA (1:200), GTSE1 (1:200), and FGL1 (1:200) overnight at $4{ }^{\circ} \mathrm{C}$. Nucleus was counterstained with DAPI (Sigma-Aldrich) and stained cells were imaged using a Zeiss Apotome (Cal Zeiss).

\section{Statistical analysis}

Data were analyzed using GraphPad Prism 5.0 (GraphPad Software Inc., San Diego, CA). Statistical difference compared with control group was determined by original oneway ANOVA with Dunnett's multiple comparisons test and Student's $t$ test; $p$ values $<0.05$ were considered significant.

\section{Results}

\section{Development of lung fibrosis after 90Gy HDFR}

Significant abnormalities were observed subsequent to the focused dose radiation in the H\&E-stained sections collected at different times (Supplementary Fig. S2a) [14]. Collagen deposition assays of Masson's trichrome staining to visualize the blue-colored collagen deposition or sirius red staining to visualize the red-colored collagen deposition after IR revealed fibrotic changes that began in the focal irradiated area, with peak collagen deposition evident 4 weeks after IR. Fibrotic changes were also observed in the tissue neighboring the focally irradiated areas of the lungs, similar to focally irradiated regions. However, fibrosis intensities were much weaker in nonirradiated neighboring tissues than focal irradiation regions. Without IR, age-related collagen deposition was not occurred in lung tissues until 4 weeks (Fig. 1a and Supplementary Fig. S2a). Hydroxyproline staining data also indicated the development of fibrosis in both focal and neighboring tissues (Supplementary Fig. S2c). The cDNA microarray data suggested that fibrosis-related genes involving mesenchymal markers, matrix metallopeptidase, fibrosis stimulatory pathways, and cytokines were changed according to fibrosis development. These phenomena were also detected in neighboring tissues (Fig. 1b and Supplementary Fig. S3). Especially, expressions of fibronectin, mmp 2, mmp 12, twist1, il1 $\beta$, and 

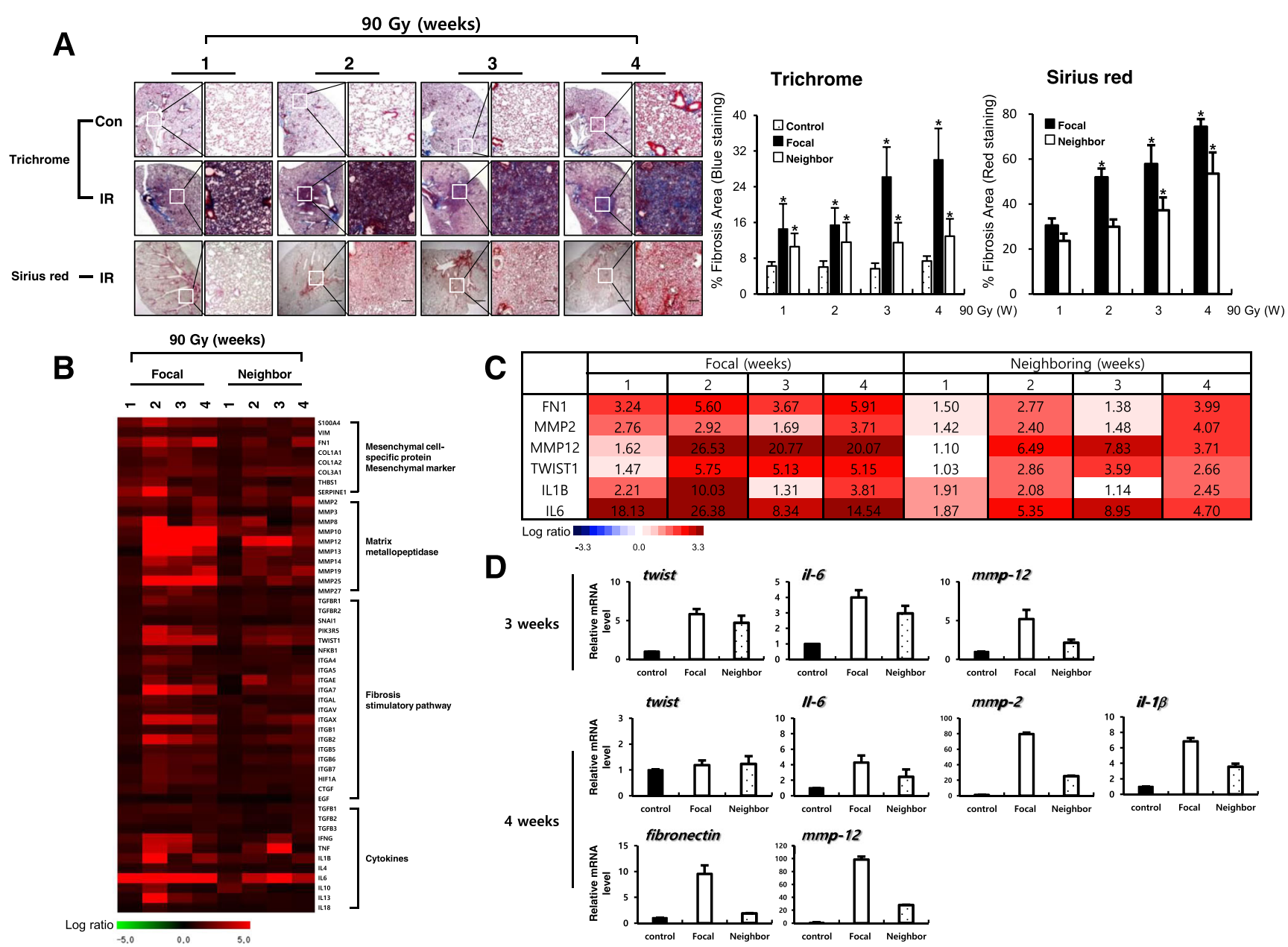

Fig. 1 Fibrotic changes of focal and neighboring regions after focal highdose radiation (90 Gy). a Masson's trichrome (upper) and Sirius red (bottom) from animals in the non-irradiated control (Con) and irradiated (90Gy IR) groups. Graphs represent quantification of fibrosis score in focal lung regions and neighboring lung regions. The arrows indicate the focally irradiated area. Magnification, $\times 1.25$ and $\times 100$; scale bar, $50 \mu \mathrm{m}(n=3$, mean $\pm \mathrm{SD}, * p<0.05$ vs. compared to the age-matched unirradiated control group (Trichrome), $* p<0.05$ vs. compared to the corresponding 1 week group (Sirius red), one-way ANOVA). b Heatmap representing the differentially expressed fibrosis-related genes by

focal high-dose radiation in both irradiated and neighboring regions at the indicated time points. Each column represents pooled lung tissue RNA samples from three mice at each time point to exclude experimental bias. The expression ratio color scale ranges from red (high) to green (low), as indicated by the scale bar with $\log 2$ units. c List of fibrosisrelated genes with a fold ratio $>2$ or $<0.5$ (for up and downregulation, respectively) compared to the control. d Quantitative RT-PCR using focal irradiated and non-irradiated neighboring lung tissue from three individual mice at the indicated time points. Each mRNA expression was normalized to gapdh $(n=3$, mean $\pm \mathrm{SD})$

il6 were markedly increased in focally irradiation regions 3 or 4 weeks after IR in microarray data. The findings were confirmed by qRT-PCR, which also correlated well in neighboring tissues (Fig. 1c).

\section{Development of lung fibrosis after 20Gy diffused whole-lung irradiation}

We also examined fibrosis development by diffused irradiation, $20 \mathrm{~Gy}(7 \mathrm{~mm})$ to the entire left lungs of mice. A significant amount of lung fibrosis was observed, with peak collagen deposition at 12 months after IR. There were more fibrotic areas following 90Gy HDFR than $20 \mathrm{~Gy}$. Interestingly, collagen deposition was also observed in non-irradiated right lung tissues after IR, although fibrosis intensity was much weaker than irradiated left lungs. Age-dependent collagen deposition was detected at later time point of 12 months; however, IR more facilitated the fibrosis development in both irradiated and non-irradiated regions (Fig. 2a and Supplementary Fig. S2b). Hydroxyproline staining data also indicated the development of fibrosis in both irradiated left lung and non-irradiated right lung tissues (Supplementary Fig. S2d). Fibrosis-related genes from the microarray data reconfirmed by qRT-PCR revealed several differences from HDFR (Fig. 2b and Supplementary Fig. S3). Only mmp12, il6, and ill3 genes were altered in both irradiated left lungs and non-irradiated right lungs (Fig. 2c). When 


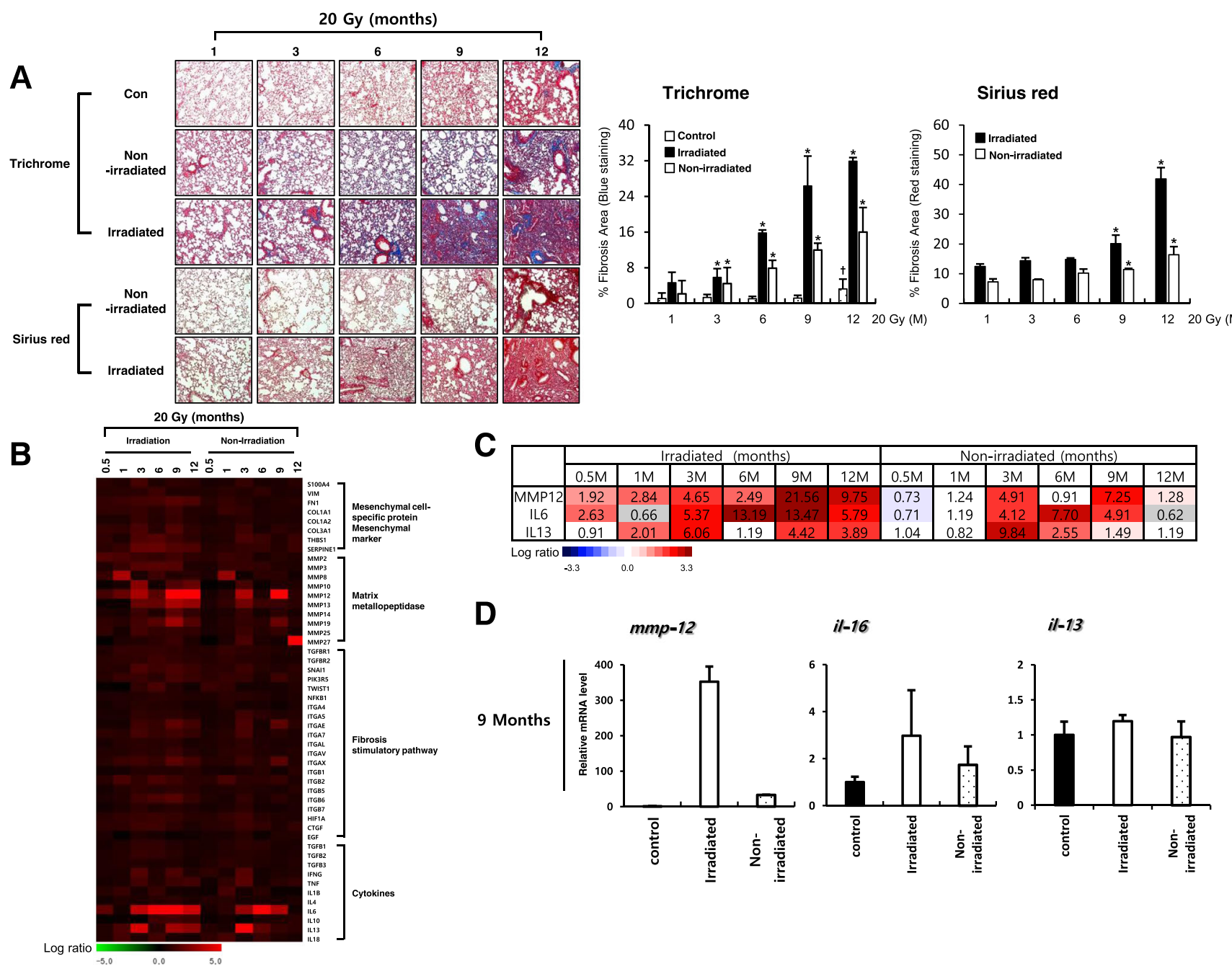

Fig. 2 Fibrotic changes of irradiated and non-irradiated regions by diffused irradiation (20 Gy). a Masson's trichrome (upper) and Sirius red (bottom) from animals in the non-irradiated control (Con) and 20Gy irradiated groups. Graphs represent quantification of fibrosis score in irradiated left lung regions and non-irradiated right lung regions. Magnification, $\times 1.25$ and $\times 100$; scale bar, $50 \mu \mathrm{m}(n=3$, mean \pm SD, ${ }^{*} p<0.05$ vs. compared to the age-matched unirradiated control group (Trichrome), $* p<0.05$ vs. compared to the corresponding 1 -week group (Sirius red), $\dagger p<0.05$ vs. compared to the 1-week unirradiated control group, one-way ANOVA). b Heat-map representing the differentially

TGF $\beta$-related gene expressions were extracted from the microarray data, no dramatic alteration was observed in both $90 \mathrm{~Gy}$ and $20 \mathrm{~Gy}$ irradiated lungs, except for slight alterations of TGF $\beta$, TGF $\beta$-receptor 1 (TGFBR1), and Smad 6 (Supplementary Fig. S4a).

\section{Selection of differentially expressed genes}

The temporal changes in gene expression of both focal irradiated and neighboring tissues following HDFR were hierarchically clustered. One week after HDFR, there were no noticeable changes in gene expression. However, at 2 and 3 weeks, expressed fibrosis-related genes by diffused irradiation in both irradiated left and non-irradiated right lungs. Each column represents pooled lung tissue RNA samples from three mice at each time point to exclude experimental bias. The expression ratio color scale ranges from red (high) to green (low), as indicated by the scale bar with $\log 2$ units. c List of fibrosis-related genes with a fold ratio $>2$ or $<0.5$ (for up and downregulation, respectively) compared to the control. d Quantitative RT-PCR using focal irradiated and non-irradiated neighboring lung tissue from three individual mice at the indicated time points. Each mRNA expression was normalized to gapdh $(n=3$, mean $\pm \mathrm{SD})$

expression of a predominant number of genes was downregulated and then restored to the control level at 4 weeks after IR. In addition to the downregulated pattern, we also identified other temporally upregulated genes, especially in the focal area of the lungs that received 90Gy IR (Fig. 3a). Interestingly, this major pattern of gene expression was also observed in the neighboring lung areas but was not observed in the lungs irradiated with 20 Gy. To identify genes responsive to 90 Gy in both focal irradiated and neighboring tissues, we selected genes presenting greater than twofold change in expression compared to the control group at every time points. The downregulated genes (fabpl, rptn, krt13, cst9, asgr1, cidec, ppp1r1b, capn13, 


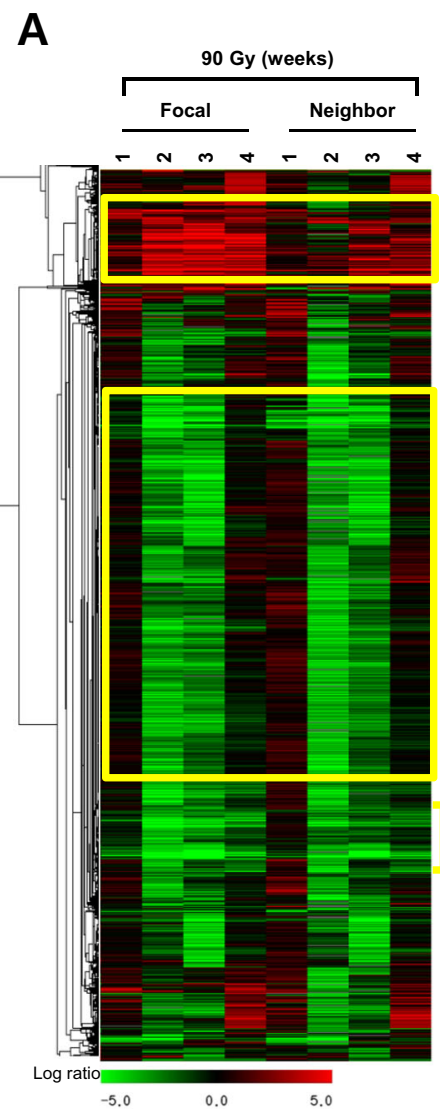

\section{B}
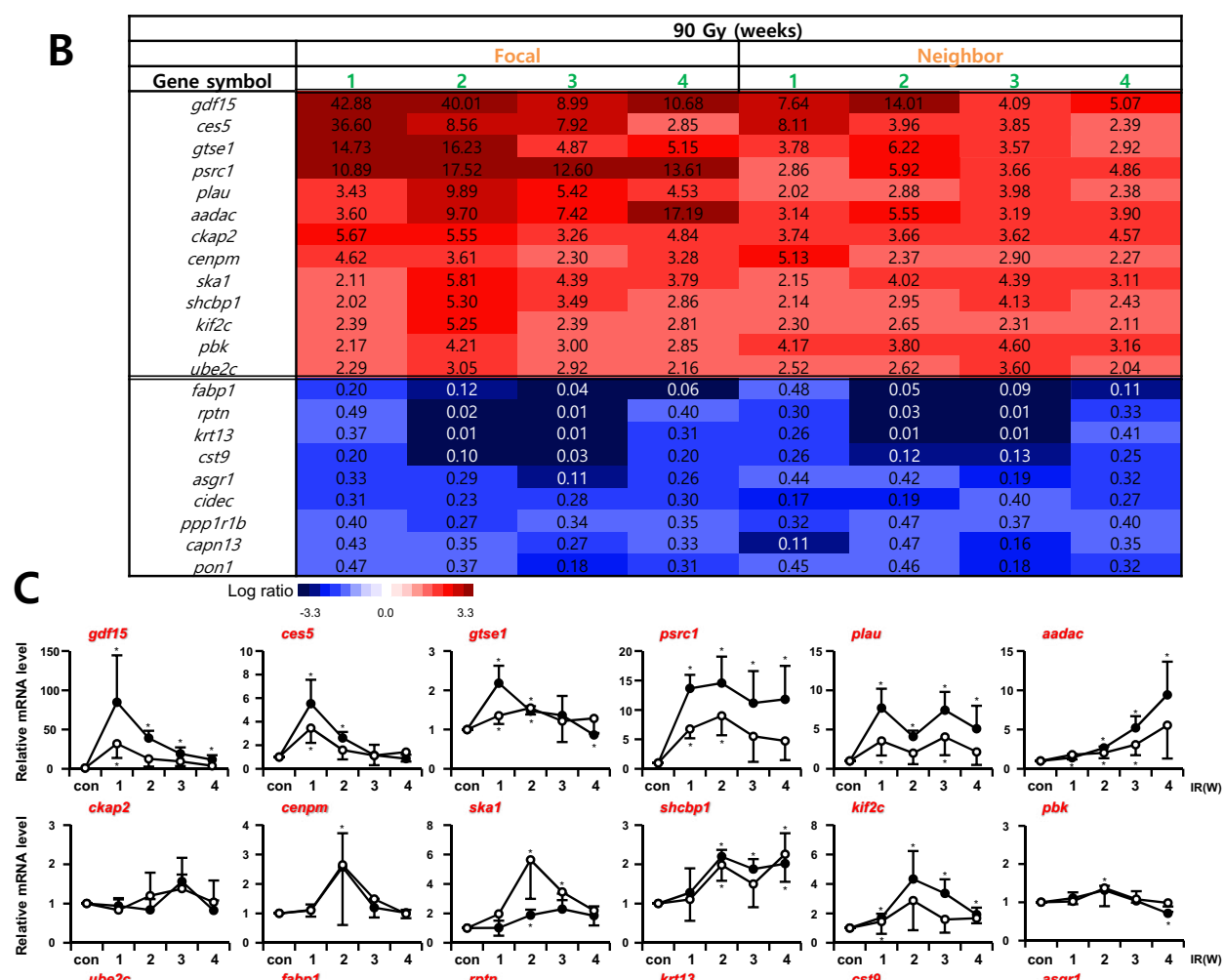

pbk


Fig. 3 Temporal gene expression patterns in focal irradiated and neighboring lung regions by $90 \mathrm{~Gy}$. a Microarray data from mouse lungs were obtained at indicated time points after exposure to $90 \mathrm{~Gy}$. The expression ratio color scale ranges from red (high) to green (low), as indicated by the scale bar with $\log 2$ units. b List of candidate genes

with a fold ratio $>2$ or $<0.5$ (for up and downregulation, respectively) compared to the control. $\mathbf{c}$ Confirmation of candidate genes by quantitative RT-PCR using the lungs from three individual mice. Each mRNA expression was normalized to gapdh $(n=3$, mean $\pm \mathrm{SD}, * p<0.05$ vs. corresponding control, one-way ANOVA)

and pon1) and upregulated genes (gdf15, ces5, gtsel, psrcl, plau, aadac, ckap2, cenpm, ska1, shcbp1, kif2c, pbk, and $u b e 2 c$ ) were selected as candidate HDFR responsive genes in both focal irradiated and neighboring tissues (Fig. 3b), which were confirmed by qRT-PCR (Fig. 3c).

We also compared temporal patterns of gene expression in diffused irradiated left lungs and non-irradiated right lung tissue with 20 Gy (Fig. 4a). Unlike the HDFR microarray data, gene expression patterns of irradiated and non-irradiated regions were not similar. When we selected more than twofold changes in expression compared to the control group at every time points, only two genes (the downregulated gene, $n g p$, and the upregulated gene, $f g l l$; the latter not observed in lungs irradiated with HDFR) were selected as candidate responsive genes for fibrosis in both 20Gy irradiated and non-irradiated lung tissue
(Fig. 4b). The results were confirmed by qRT-PCR (Fig. 4c). Gene of gtse1, selected from the 90Gy irradiated lung tissues, and gene of fgll, selected from 20Gy irradiated lung tissues, were also overexpressed in microarray data of other dose irradiated lungs, although the intensity was weaker (Supplementary Fig. S4b), suggesting both genes are regarded as IR-responsible genes regardless of IR doses.

\section{Genes responsible for IR-induced epithelial-mesenchymal transition (EMT)}

To determine the genes responsible for IR-induced lung fibrosis, siRNA of each gene that showed relatively higher expression or lower expression in microarray data was transfected to L132 cells and RT-PCR analysis for 


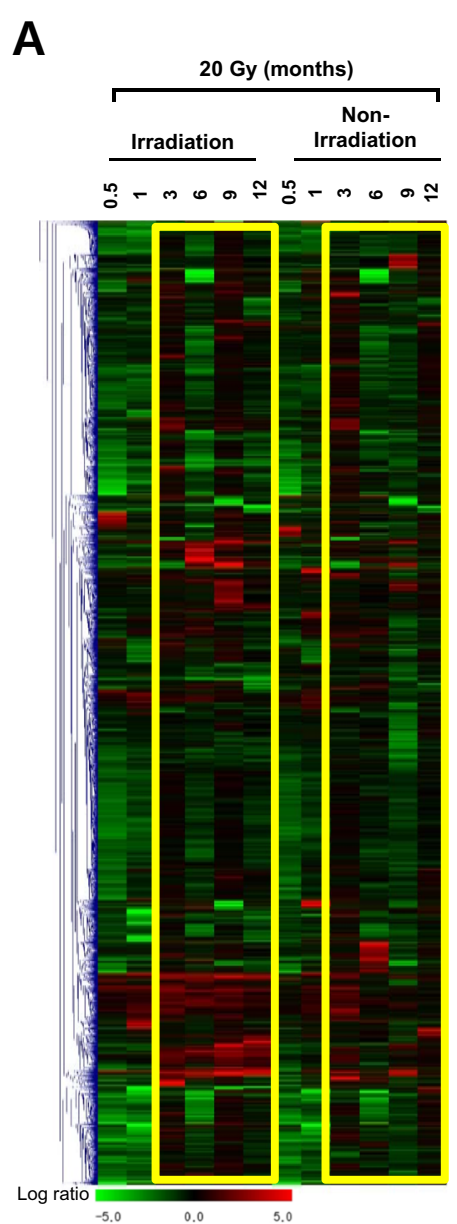

Fig. 4 Temporal gene expression patterns in irradiated and non-irradiated lung tissue by diffused irradiation ( $20 \mathrm{~Gy}$ ). a Microarray data from mouse lungs were obtained at indicated time points after exposure to $20 \mathrm{~Gy}$. The expression ratio color scale ranges from red (high) to green (low), as indicated by the scale bar with $\log 2$ units. $\mathbf{b}$ List of candidate genes with

EMT-related genes such as E-cadherin and snail was performed after treatment of TGF $\beta$ (data not shown). The gtsel and fgll were identified as candidate genes from the results, and these two genes (both mRNA and protein) responded to both IR ( $8 \mathrm{~Gy})$ and TGF $\beta(5 \mathrm{ng} / \mathrm{ml})$, accompanied with increased Snail, Twist, MMP12 and Fibronectin, and reduction of ZO-1 (Fig. 5a and Supplementary Fig. S5a). Altered expression of EMT markers by IR or TGF $\beta$ was partially restored in terms of protein level and mRNA levels of L132 normal lung cells (Fig. 5b and Supplementary Fig. S5b). TGF $\beta$ mediated morphological changes were restored by siRNA of gste1 or fgll (Supplementary Fig. S5c). The results suggested the involvement of Gstel and Fgll in IR or TGF $\beta$-mediated EMT. Co-expression of Fibronectin or Twist with Gtse1 or Fgl1 after IR or TGF $\beta$ treatment suggested the involvement of these genes in EMT processing (Fig. 5c and Supplementary Fig. S5d). a fold ratio $>2$ or $<0.5$ (for up and downregulation, respectively) compared to the control. c Confirmation of candidate genes by quantitative RT-PCR using lungs from three individual mice. Each mRNA expression was normalized to gapdh $(n=3$, mean $\pm \mathrm{SD}, * p<0.05$ vs. corresponding control, one-way ANOVA)

Moreover, knockdown of gtse 1 or fgl1 inhibited IR or TGF $\beta$-mediated migration (Fig. 5d and Supplementary Fig. S5e) even though knockdown of gtse1 or fgll did not induce any increased cell death after IR or TGF $\beta$ (Supplementary Fig. S6).

\section{Overexpression of Gtse1 and Fgl1 in IR-induced fibrotic lung tissues}

Immunofluorescence data of GTSE1 and FGL1 with costaining of $\alpha$-SMA demonstrated the increased expression in fibrotic regions of lung tissues after 20 Gy or 90 Gy. Gtse1 expression in accompanied with higher expression of $\alpha$-SMA was clearly shown in non-irradiated neighboring tissues, as well as 90Gy focal irradiated regions, when compared with the age-matched unirradiated control mice at 2 and 4 weeks after IR. Moreover, its increased expression was also observed in the case of 20Gy. Fgll expression was dominant in 20Gy 

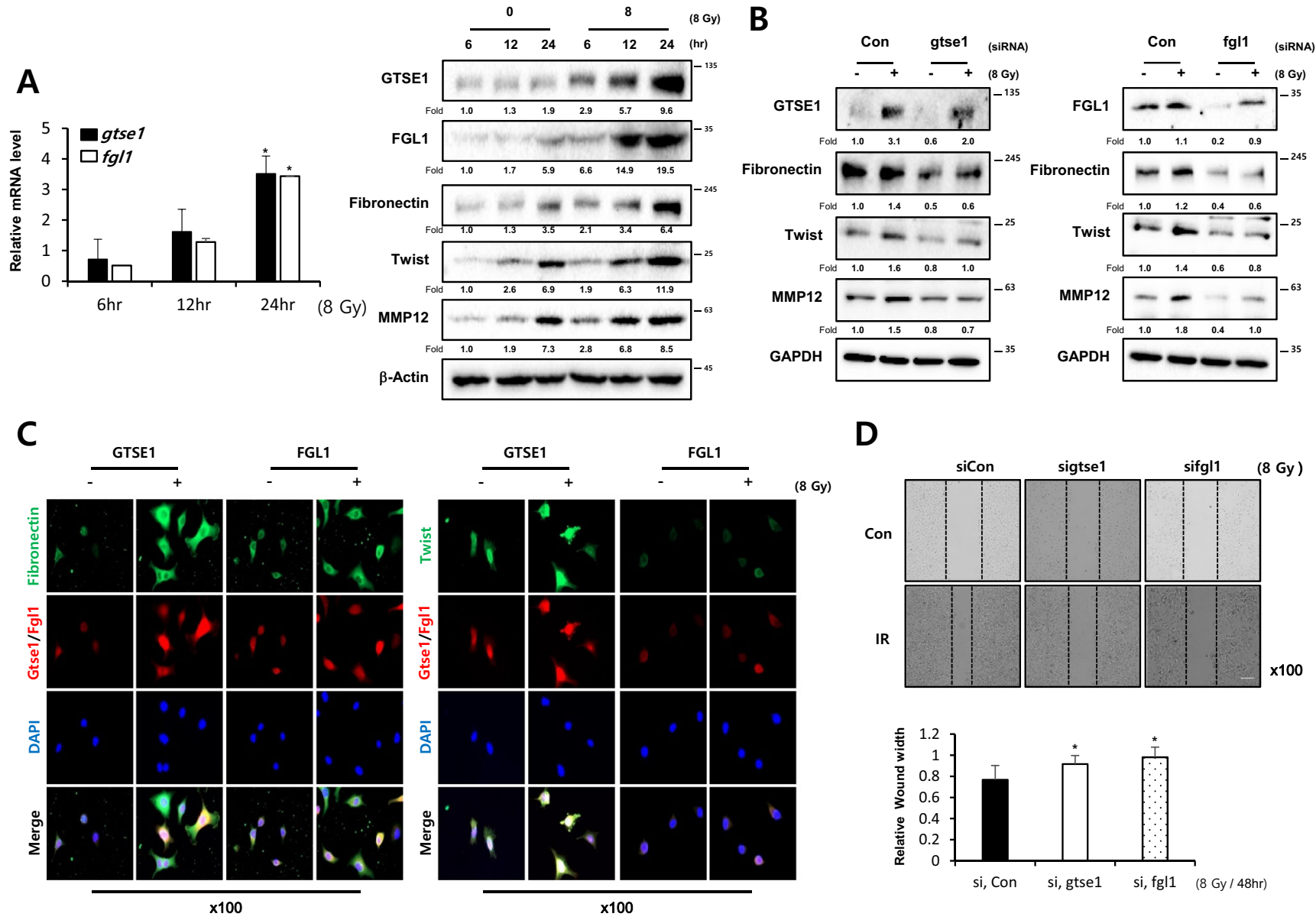

C GTSE1 and FGL1 in L132 cells was examined by qRT-PCR (left) and Western blotting (right) analysis after 8 Gy radiation (IR) at indicated time points. b Western blot analysis in L132 cells after transfection of siRNA gtsel or $f g l l$ with or without $8 \mathrm{~Gy}$ radiation (IR). The fold increase of each time point mRNA was normalized with the non-irradiated cells $(n=3$, mean $\pm \mathrm{SD}, * p<0.05$ vs. corresponding control, paired sample $t$ test) and protein levels were quantified using Image $J$ software, and data are expressed as the fold change relative to the negative control. c Co- immunofluorescence staining of Fibronectin or Twist (green) and GTSE1 (red) or FGL1 (red) after $24 \mathrm{~h}$ of irradiation (8 Gy) in A549 cells. Nuclei were counterstained with DAPI (blue). $\mathbf{d}$ Wound healing assays in A549 cells after transfection of siRNA gtsel or $f g l l$ with or without irradiation ( $8 \mathrm{~Gy}$ ). Cell movement into wound was shown at $48 \mathrm{~h}$ postscratch. Graphs represent relative wound width (mean $\pm \mathrm{SD}, * p<0.05$ vs. corresponding control, paired sample $t$ test). All representative photomicrographs magnification, $\times 100$; bar, $50 \mu \mathrm{m}$ irradiated left lung tissues and in non-irradiated right lungs, when compared with the age-matched unirradiated control mice. IR with 90 Gy also increased Fgl1 expression in both focal irradiated and neighboring tissues (Fig. 6a and b).

\section{Discussion}

Our results using SBRT mouse model demonstrate that nonirradiated neighboring regions had a similar gene expression pattern to the pattern observed in the focally irradiated lung regions. Moreover, visual confirmation and changes in gene expression provide evidence of the development of fibrosis even in non-irradiated neighboring lung regions. The fibrotic changes of the boundary regions were weaker than the focally irradiated area of the lung. Moreover, fibrosis-related genes identified in the focally irradiated areas were similarly evident in neighboring non-irradiated lung regions. Comparison to the fibrosis after the left lung diffused 20Gy irradiation revealed that the development of fibrosis in the non-irradiated right lung was also affected, even though the potency was much weaker than HDFR.

Our animal models reflect focal and high-dose irradiation of human SBRT. This study is a first trial for the investigation of SBRT-related gene expression patterns in mice during lung fibrosis. Because lung fibrosis by IR was mainly in neighboring regions of irradiated tumor tissues, we also investigated nonirradiated neighboring lungs. Down and upregulated genes were identified, which regarded as candidate HDFR responsive genes because of their similar expression patterns in focally 
A

GTSE1

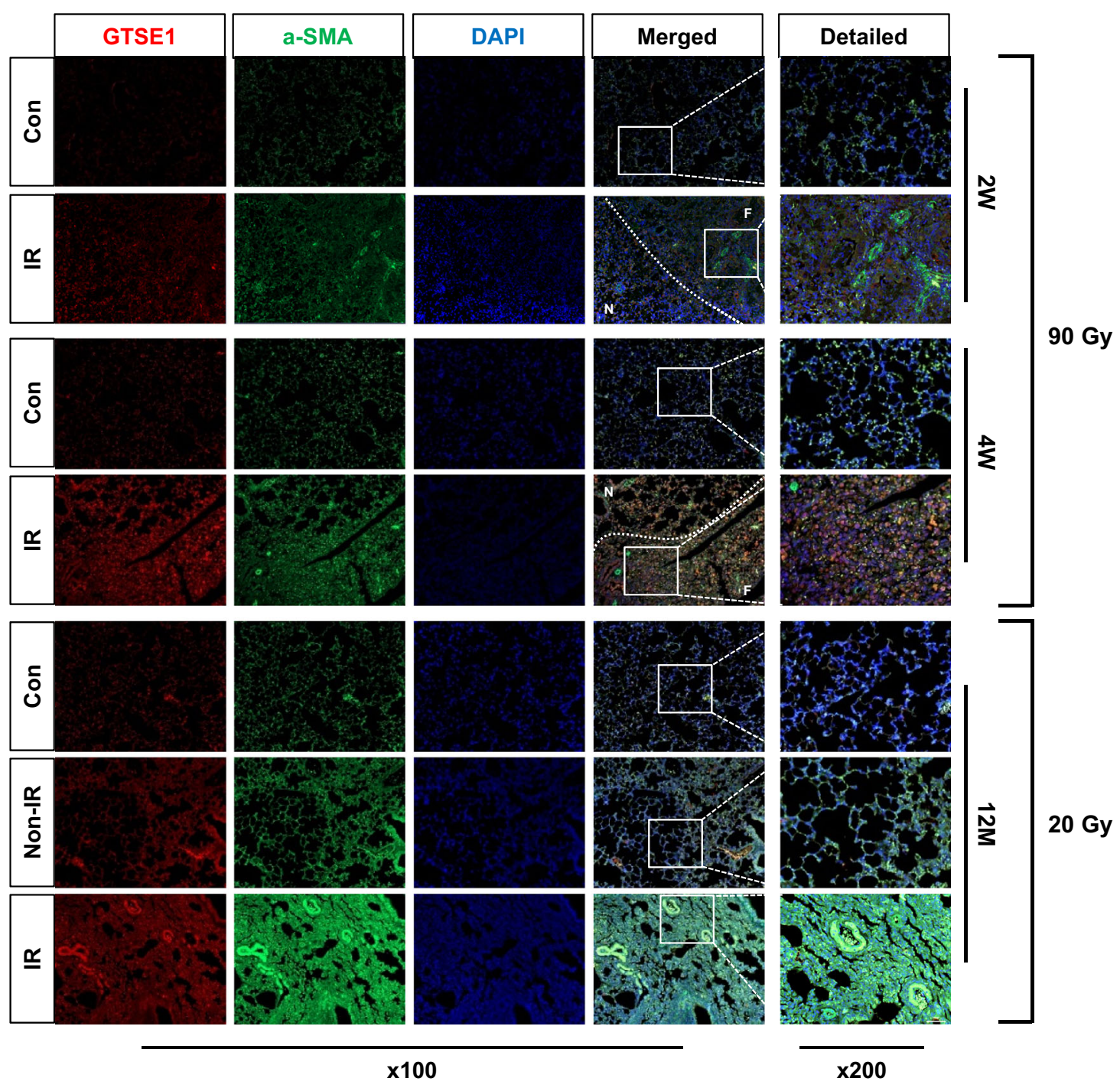

Fig. 6 Overexpression of GTSE1 or FGL1 in IR-induced lung fibrotic tissues. a, b Co-immunofluorescence staining of $\alpha$-SMA (green) and GTSE1/FGL1 (red) after focal high-dose (90 Gy) and diffused whole- lung irradiation $(20 \mathrm{~Gy})$ at each time point. Lung tissue sections from three mice were used. Magnification, $\times 100$ and $\times 200$; scale bar, $50 \mu \mathrm{m}$. (F: focal irradiated region, $\mathrm{N}$ : neighboring region) irradiated and neighboring regions. When we compared the gene expression by 20Gy diffused irradiation, two genes responded similarly in both irradiated left lung and nonirradiated right lung. When we screened using HDFR responsive genes and 20Gy responded genes to ascertain whether they were involved in IR or TGF $\beta$-mediated EMT in cell system, gtsel and $f g l l$ were identified as candidate genes for regulation of EMT. GTSE1, which was identified from 90Gy focal irradiated lungs, is specifically expressed during S and G2 phases of the cell cycle $[15,16]$. It is a microtubule-associated protein that interacts with microtubules or PLK1 [17]. It also responds to DNA damage and inhibits apoptosis. GTSE1 inhibition significantly decreased tumor growth in vitro and in vivo, and suppressed migration and invasion in vitro [18]. Our costaining data of GTSE1 and Ki67 revealed that fibrotic regions showed high expression of Ki67 accompanied with GTSE1 overexpression in $90 \mathrm{~Gy}$ focal irradiated lung tissues (Supplementary Fig. S7). The $f g l l$ (fibrinogen-like protein 1 [FREP1], also termed hepassocin) was identified from 20Gy diffused irradiated lungs. FGL1 is a hepatocyte secreted protein containing a fibrinogen-related domain in its C-terminal portion [19]. The enhancement of FGL1 levels was regulated by IL-6 $[20,21]$ and it participates in the development of non-alcoholic fatty liver disease, hepatocellular carcinomas, and hepatocyte mitogenic activity [22-26]. However, there are no reports of the involvement of any of these genes in EMT or fibrosis. This 
B

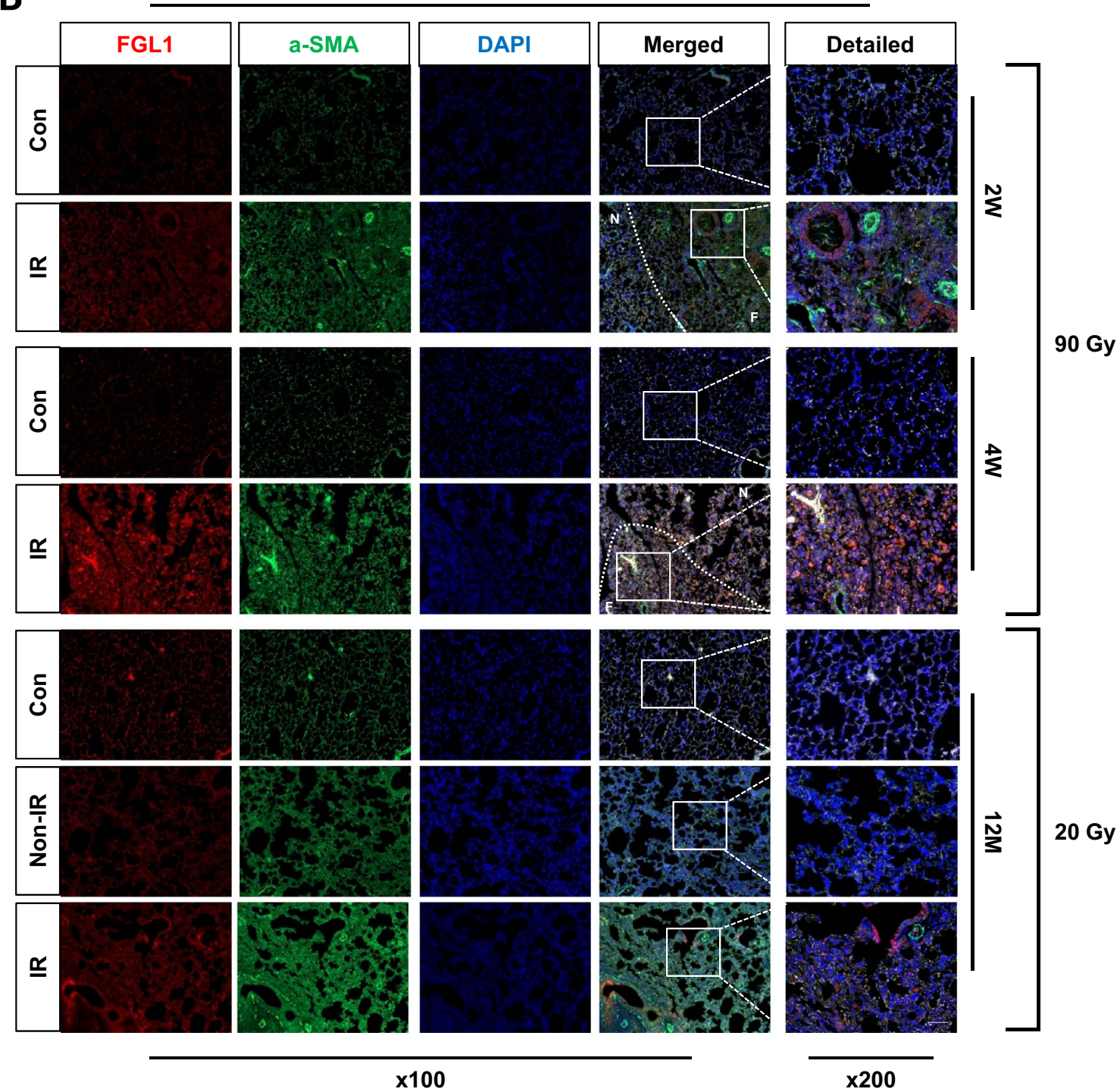

Fig. 6 continued.

study for the first time suggests GTSE1 and FGL1 as regulators of EMT and cellular migration.

HDFR and low-dose diffused radiation showed different fibrosis development and molecular events and regulators may not be same. GTSE1 was selected from 90Gy HDFR lung and FGL1 from diffused irradiated lung. However, in vitro cell system, these two genes were all responded (showed the increased expression) by both IR and TGF $\beta$, suggesting involvement in common fibrosis pathways regardless of fibrosis inducers. Moreover, immunostaining using lung tissues indicated that GTSE1 and FGL1 were increased at fibrotic regions regardless of focal high-dose and diffused irradiation. Since IR slightly induced mRNA of TGF $\beta$, TGFBR1, and Smad 6 especially in 90Gy irradiated lungs, TGF $\beta$ mediated direct or indirect EMT mechanisms by IR may involve in expression of these two gene expressions during lung fibrosis. Another interesting finding is that upregulation of GTSE1 and FGL1 was in the early time points of fibrosis. Immunofluorescence data also indicated that surrounding tissues of fibrotic regions showed overexpression of GTSE1 and FGL1, as well as irradiated fibrotic regions in mouse lungs, suggesting that upregulation of GTSE1 and FGL1 may occur during the process of going to fibrosis, but it is not an outcome.

In conclusion, we established a mouse irradiation system and identified the molecular signatures for IRinduced lung fibrosis. Although further functional analyses are required to determine the roles of GTSE1 or FGL1 during development of lung fibrosis, our results might provide the information that is useful for understanding IR-induced lung fibrosis. 
Funding information This work was supported by grants from the National Research Foundation of Korea (NRF-2017R1A2B2002327, NRF-2017M2A2A702019560, and 2018R1A5A2025286), funded by the Korean government (Ministry of Science and ICT).

\section{Compliance with ethical standards}

Conflict of interest The authors declare that they have no conflict of interest.

All animal experiments were approved by the Yonsei University Health System-Institutional Animal Care and Use committee (YUHSIACUC; 2015-0267) and were performed in accordance with the relevant guidelines and regulations.

Open Access This article is distributed under the terms of the Creative Commons Attribution 4.0 International License (http:// creativecommons.org/licenses/by/4.0/), which permits unrestricted use, distribution, and reproduction in any medium, provided you give appropriate credit to the original author(s) and the source, provide a link to the Creative Commons license, and indicate if changes were made.

\section{References}

1. Liu H (2017) Plasminogen activator inhibitor-1 gene is associated with radioactive lung injury in lung cancer patients. Biomed Res 28:6541-6545

2. Kim H, Bae H, Lee MY, Cheong KH, Kim KJ, Han T, Kang SK, Park S, Hwang T, Yoon JW, Kim LS (2014) Analysis of predictive factors for lung injury after forward-planned intensity-modulated radiotherapy in whole breast irradiation. J Breast Cancer 17:69-75

3. Williams JP, Johnston CJ, Finkelstein JN (2010) Treatment for radiation-induced pulmonary late effects: spoiled for choice or looking in the wrong direction? Curr Drug Targets 11:1386-1394

4. Fu XL, Huang H, Bentel G, Clough R, Jirtle RL, Kong FM, Marks LB, Anscher MS (2001) Predicting the risk of symptomatic radiation-induced lung injury using both the physical and biologic parameters V(30) and transforming growth factor beta. Int J Radiat Oncol Biol Phys 50:3212899-3212908

5. Roach M 3rd, Gandara DR, Yuo HS, Swift PS, Kroll S, Shrieve DC, Wara WM, Margolis L, Phillips TL (1995) Radiation pneumonitis following combined modality therapy for lung cancer: analysis of prognostic factors. J Clin Oncol 13:2606-2612

6. Cho J, Kodym R, Seliounine S, Richardson JA, Solberg TD, Story MD (2010) High dose-per-fraction irradiation of limited lung volumes using an image-guided, highly focused irradiator: simulating stereotactic body radiotherapy regimens in a small-animal model. Int J Radiat Oncol Biol Phys 77:895-902

7. Jin H, Jeon S, Kang GY, Lee HJ, Cho J, Lee YS (2017) Identification of radiation response genes and proteins from mouse pulmonary tissues after high-dose per fraction irradiation of limited lung volumes. Int J Radiat Biol 93:184-193

8. Xiong S, Pan X, Xu L, Yang Z, Guo R, Gu Y, Li R, Wang Q, Xiao F, Du L, Zhou P, Zhu M (2018) Regulatory T cells promote $\beta$ catenin-mediated epithelium-to-mesenchyme transition during radiation-induced pulmonary fibrosis. Int J Radiat Oncol Biol Phys 93:425-435

9. Wang J, Zhou F, Li Z, Mei H, Wang Y, Ma H, Shi L, Huang A, Zhang T, Lin Z, Wu G (2018) Pharmacological targeting of BET proteins attenuates radiation-induced lung fibrosis. Sci Rep 8:998
10. Zhang C, Zhao H, Li BL, Fu-Gao LH, Cai JM, Zheng M (2018) CpG-oligodeoxynucleotides may be effective for preventing ionizing radiation induced pulmonary fibrosis. Toxicol Lett 292:181-189

11. Wei W, Zhang HY, Gong XK, Dong Z, Chen ZY, Wang R, Yi JX, Shen YN, Jin SZ (2018) Mechanism of MEN1 gene in radiationinduced pulmonary fibrosis in mice. Gene 678:252-260

12. Hong ZY, Eun SH, Park K, Choi WH, Lee JI, Lee EJ, Lee JM, Story MD, Cho J (2014) Development of a small animal model to simulate clinical stereotactic body radiotherapy-induced central and peripheral lung injuries. J Radiat Res 55:648-657

13. Al-Dasooqi N, Bowen JM, Gibson RJ, Logan RM, Stringer AM, Keefe DM (2011) Irinotecan-induced alterations in intestinal cell kinetics and extracellular matrix component expression in the Dark Agouti rat. Int J Exp Pathol 92:357-365

14. Hong ZY, Lee HJ, Choi WH, Lee YJ, Eun SH, Lee JI, Park K, Lee JM, Cho J (2014) A preclinical rodent model of acute radiationinduced lung injury after ablative focal irradiation reflecting clinical stereotactic body radiotherapy. Radiat Res 182:83-91

15. Utrera R, Collavin L, Lazarević D, Delia D, Schneider C (1998) A novel p53-inducible gene coding for a microtubule-localized protein with G2-phase-specific expression. EMBO J 17:5015-5025

16. Monte M, Collavin L, Lazarevic D, Utrera R, Dragani TA, Schneider C (2000) Cloning, chromosome mapping and functional characterization of a human homologue of murine gtse-1 (B99) gene. Gene 254:229-236

17. Liu XS, Li H, Song B, Liu X (2010) Polo-like kinase 1 phosphorylation of G2 and S-phase-expressed 1 protein is essential for $\mathrm{p} 53$ inactivation during G2 checkpoint recovery. EMBO Rep 11:626632

18. Guo L, Zhang S, Zhang B, Chen W, Li X, Zhang W, Zhou C, Zhang J, Ren N, Ye Q (2016) Silencing GTSE-1 expression inhibits proliferation and invasion of hepatocellular carcinoma cells. Cell Biol Toxicol 32:263-274

19. Yamamoto T, Gotoh M, Sasaki H, Terada M, Kitajima M, Hirohashi S (1993) Molecular cloning and initial characterization of a novel fibrinogen-related gene, HFREP-1. Biochem Biophys Res Commun 193:681-687

20. Liu Z, Ukomadu C (2008) Fibrinogen-like protein 1, a hepatocyte derived protein is an acute phase reactant. Biochem Biophys Res Commun 365:729-734

21. Xagorari A, Siotou E, Yiangou M, Tsolaki E, Bougiouklis D, Sakkas L, Fassas A, Anagnostopoulos A (2013) Protective effect of mesenchymal stem cell-conditioned medium on hepatic cell apoptosis after acute liver injury. Int J Clin Exp Pathol 6:831-840

22. Wu HT, Lu FH, Ou HY, Su YC, Hung HC, Wu JS, Yang YC, Wu CL, Chang CJ (2013) The role of hepassocin in the development of non-alcoholic fatty liver disease. J Hepatol 59:1065-1072

23. Cao MM, Xu WX, Li CY, Cao CZ, Wang ZD, Yao JW, Yu M, Zhan YQ, Wang XH, Tang LJ, Chen H, Li W, Ge CH, Yang XM (2011) Hepassocin regulates cell proliferation of the human hepatic cells L02 and hepatocarcinoma cells through different mechanisms. J Cell Biochem 112:2882-2890

24. Hara H, Yoshimura H, Uchida S, Toyoda Y, Aoki M, Sakai Y, Morimoto S, Shiokawa K (2001) Molecular cloning and functional expression analysis of a cDNA for human hepassocin, a liverspecific protein with hepatocyte mitogenic activity. Biochim Biophys Acta 1520:45-53

25. Gao M, Zhan YQ, Yu M, Ge CH, Li CY, Zhang JH, Wang XH, Ge ZQ, Yang XM (2014) Hepassocin activates the EGFR/ERK cascade and induces proliferation of L02 cells through the Srcdependent pathway. Cell Signal 26:2161-2166

26. Demchev V, Malana G, Vangala D, Stoll J, Desai A, Kang HW, Li Y, Nayeb-Hashemi H, Niepel M, Cohen DE et al (2013) Targeted deletion of fibrinogen like protein 1 reveals a novel role in energy substrate utilization. PLoS One e58084:8 